\title{
Alterstice
}

Revue internationale de la recherche interculturelle

International Journal of Intercultural Research

Revista International de la Investigacion Intercultural

\section{Pluralité des langues et des cultures : quatre expériences institutionnelles, une même quête d'équilibre dans la tension entre égalité et diversité}

\section{Tania Ogay}

Volume 2, numéro 1, 2012

Stratégies de (re)médiation en situation plurilingue. Études de cas en contextes de recherche et d'éducation

URI : https://id.erudit.org/iderudit/1077549ar

DOI : https://doi.org/10.7202/1077549ar

Aller au sommaire du numéro

Éditeur(s)

Alterstice

ISSN

1923-919X (numérique)

Découvrir la revue

Citer cet article

Ogay, T. (2012). Pluralité des langues et des cultures : quatre expériences institutionnelles, une même quête d'équilibre dans la tension entre égalité et diversité. Alterstice, 2(1), 9-16. https://doi.org/10.7202/1077549ar

\section{Résumé de l'article}

Comment articuler pluralité des langues et des cultures ? Comment assurer la communication entre des interactants aux langues premières et aux références culturelles différentes ? Trois contextes institutionnels pratiquant le bilinguisme selon la stratégie de l'intercompréhension sont discutés en regard des questions posées par un quatrième contexte, une association scientifique internationale qui a adopté la stratégie de la communication via une lingua franca, en l'occurrence le français. La comparaison et la discussion de ces quatre contextes montre la distance qu'il peut y avoir entre les discours prônant la diversité et les actes des individus dans les situations d'interactions. La communication plurilingue est exigeante et demande des efforts importants et constants, de l'institution comme des individus censés la pratiquer au quotidien. Communiquer grâce à une lingua franca apparaît comme une solution réaliste lorsque les moyens sont limités. Il convient cependant d'expliciter le statut de la langue choisie comme langue de communication, en la déshabillant de sa fonction d'expression et detransmission d'une culture pour la limiter à sa fonction instrumentale. 


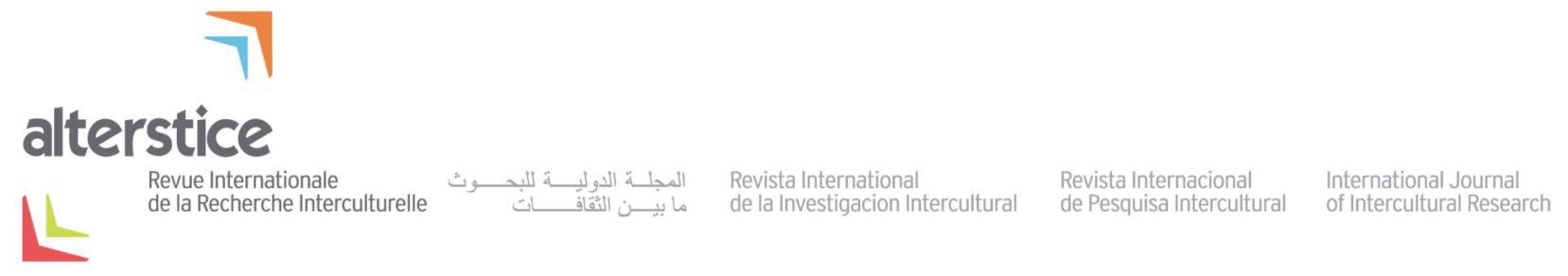

ARTICLE THÉMATIQUE

\section{Pluralité des langues et des cultures : quatre expériences institutionnelles, une même quête d'équilibre dans la tension entre égalité et diversité}

Tania Ogay ${ }^{1}$

\section{Résumé}

Comment articuler pluralité des langues et des cultures? Comment assurer la communication entre des interactants aux langues premières et aux références culturelles différentes? Trois contextes institutionnels pratiquant le bilinguisme selon la stratégie de l'intercompréhension sont discutés en regard des questions posées par un quatrième contexte, une association scientifique internationale qui a adopté la stratégie de la communication via une lingua franca, en l'occurrence le français. La comparaison et la discussion de ces quatre contextes montre la distance qu'il peut y avoir entre les discours prônant la diversité et les actes des individus dans les situations d'interactions. La communication plurilingue est exigeante et demande des efforts importants et constants, de l'institution comme des individus censés la pratiquer au quotidien. Communiquer grâce à une lingua franca apparaît comme une solution réaliste lorsque les moyens sont limités. II convient cependant d'expliciter le statut de la langue choisie comme langue de communication, en la déshabillant de sa fonction d'expression et de transmission d'une culture pour la limiter à sa fonction instrumentale.

\section{Rattachement de l'auteure \\ ${ }^{1}$ Département des Sciences de l'éducation, Université de Fribourg, Fribourg, Suisse}

\section{Correspondance}

tania.ogay@unifr.ch

\section{Mots clés}

plurilinguisme; bilinguisme; intercompréhension; Suisse; associations; dialectique

\section{Pour citer cet article :}

Ogay, T. (2012). Pluralité des langues et des cultures : quatre expériences institutionnelles, une même quête d'équilibre dans la tension entre égalité et diversité. Alterstice, 2(1), 9-16. 


\section{Introduction}

Après un certain nombre d'années d'activité dans le domaine de la recherche et de la formation interculturelles, il m'arrive malgré tout encore souvent d'expérimenter la difficulté de la communication interculturelle, d'autant plus lorsque mon interlocuteur et moi devons jongler avec plusieurs langues. Mais est-ce vraiment "d'autant plus »? En quoi est-il avantageux d'utiliser une même langue dans une interaction alors qu'on ne partage pas les mêmes références culturelles? II y a peut-être aussi des pièges? Comment la diversité culturelle s'articule-t-elle avec la diversité linguistique et de quelles solutions disposent les individus et les institutions pour gérer ces deux dimensions de diversité? Avec quels résultats du point de vue de l'efficacité de la communication?

En toute logique, la combinaison dans une situation d'interaction de la dimension de la diversité des langues avec celle de la diversité des cultures donne quatre configurations :

- Les interactants sont reconnus comme partageant les mêmes références culturelles et utilisent la même langue dans l'interaction. Nous nous trouvons ici dans la configuration - probablement mythique - de la communication intraculturelle.

- Les interactants sont reconnus comme partageant les mêmes références culturelles et utilisent des langues différentes dans l'interaction. II s'agit ici d'une configuration difficile à se représenter a priori, tant le lien entre langue et culture est fort et marque les esprits. Pourtant, cette configuration existe et correspond notamment aux États plurilingues, comme la Suisse. Cette configuration nous rappelle la complexité de la culture qui ne peut être pensée sur un seul niveau, reliant de façon univoque et exclusive l'appartenance à une communauté linguistique à l'identité culturelle « correspondante».

- Les interactants sont reconnus comme ayant des références culturelles différentes et utilisent la même langue dans l'interaction. De plus en plus fréquente dans le monde globalisé actuel, cette combinaison doit encore être précisée: la langue utilisée dans l'interaction est-elle une langue étrangère pour tous les interactants (par exemple dans une réunion internationale réunissant des locuteurs de langues diverses mais non anglophones, qui choisissent cependant l'anglais comme langue commune), ou alors la langue de communication est-elle une langue étrangère seulement pour une partie d'entre eux (dans cette réunion internationale, certains participants viennent de pays anglophones) ? Ceux pour qui la langue de communication est une langue étrangère sont-ils majoritaires ou minoritaires dans la situation d'interaction ?

- Les interactants sont reconnus comme ayant des références culturelles différentes et utilisent des langues différentes dans l'interaction : cette combinaison représente le "tout pluri », le pari extrême de la diversité. Un parti tentant pour qui travaille dans «l'interculturel », mais à quel prix?

Pour examiner le potentiel de ces différentes combinaisons entre diversité des langues et diversité des cultures, quatre contextes institutionnels seront présentés et discutés. II ne s'agit pas là du fruit d'observations systématiques réalisées dans cette intention mais de mon expérience personnelle, à différents moments de mon parcours personnel et intellectuel. Chacun de ces contextes m'a permis en son temps d'avancer dans ma réflexion. Les exposer ensemble ici me permet de porter un regard critique sur les solutions qui sont prônées pour articuler diversité des langues et des cultures, et ainsi de mieux saisir les enjeux des choix linguistiques qui se posent actuellement dans le dernier contexte analysé.

\section{Quatre contextes institutionnels aux prises avec la diversité des langues et des cultures}

Premier cas : une Université, au bilinguisme revendiqué

L'Université de Fribourg, en Suisse, est située à la " frontière des langues » entre l'allemand et le français, les deux langues nationales les plus parlées de Suisse (selon les dernières données disponibles, datant du recensement de 2000, $64 \%$ de la population déclarait avoir l'allemand comme langue principale, $20 \%$ le français, $6 \%$ l'italien, $1 \%$ le romanche - la seule langue qui ne soit pas partagée avec un pays voisin - et $9 \%$ d'autres langues ${ }^{1}$ ). II serait trop

${ }^{1}$ http://www.bfs.admin.ch/bfs/portal/fr/index/themen/01/05/blank/key/sprachen.html 
long ici de présenter comment la Suisse gère son plurilinguisme (voir par exemple Gohard-Radenkovic, 2007; Grin, 2010). Je ne mentionnerai que le principe de territorialité des langues, qui fait correspondre un territoire à une langue et définit ainsi la langue qui est utilisée par les institutions publiques, au nombre desquelles les institutions chargées de l'éducation et de la formation. Ainsi, lorsque l'Université de Fribourg se qualifie avec fierté de "bilingue ", elle fait référence au bilinguisme de l'institution qui, se trouvant à la frontière entre le territoire francophone (Suisse romande) et le territoire germanophone (Suisse alémanique), fonctionne avec deux langues officielles, l'allemand et le français. Elle ne se réfère pas au bilinguisme (voire plus, ou moins) des individus, qui ne recouvre pas nécessairement le bilinguisme institutionnel, tant s'en faut. Comme cela est habituellement pratiqué en Suisse au niveau fédéral, la communication est gérée selon le principe de l'intercompréhension (Capucho, 2008), plus précisément selon le « modèle suisse d'intercompréhension » (Lüdi, 2006, p. 183) : chacun doit pouvoir parler sa langue (sous-entendu, les langues officielles uniquement) et comprendre celle de l'autre. Toute personne travaillant à l'Université de Fribourg est ainsi censée avoir une maîtrise suffisante des deux langues officielles (pour les étudiants, cela peut être très différent selon les disciplines qui permettent souvent des études dans une seule langue). Chaque document institutionnel doit exister en version française et en version allemande. II n'existe pourtant à ce jour encore aucun service de traduction à disposition des collaborateurs, ce qui reviendrait à admettre que le bilinguisme de l'institution n'est pas nécessairement celui de ses acteurs.

Après quelques années dans cette institution, il m’apparaît que le bilinguisme de l'Université de Fribourg est avant tout un bilinguisme institutionnel revendiqué, considéré comme allant de soi, mais pas vraiment un bilinguisme réfléchi et pleinement réalisé. II serait d'ailleurs intéressant de procéder à une observation systématique des modes de travail des employés de l'Université afin de voir si ce fonctionnement en deux langues n'entraîne pas des réticences à la collaboration avec des collègues de l'autre groupe linguistique, des fonctionnements en parallèle alors qu'ils auraient pu ou dû être concertés. II est par ailleurs étonnant de voir combien il est peu fait référence au bilinguisme lorsqu'il s'agit de comprendre les raisons d'un conflit ou d'un projet qui ne produit pas les résultats escomptés.

Si la question de l'utilisation de deux langues différentes est rarement thématisée lorsqu'il s'agit d'analyser des situations de communication, on se doute que la dimension interculturelle l'est encore moins. L'Université de Fribourg est ici la digne fille de la Confédération helvétique, qui envisage sa diversité uniquement sous l'angle linguistique : il n'est question que de groupes linguistiques et de compréhension interlinguistique, au grand jamais de groupes culturels et d'interculturalité. Comme le note aussi Gohard-Radenkovic (2007), la dimension culturelle et interculturelle n'est réservée en Suisse qu'aux communautés immigrées. Cette perception en termes de seuls groupes linguistiques ne manque jamais d'étonner mes collègues canadiens francophones qui quant à eux n'hésitent pas à se présenter comme un groupe " ethnolinguistique ", voire même " ethnique " (voir par exemple Duquette, 2004), des termes qui choqueraient en Suisse. Bien que tous deux des États fédéraux et multilingues, le Canada et la Suisse représentent des contextes très différents, aux sensibilités à la dimension culturelle et aux revendications identitaires très différentes. Ainsi, en Suisse et à l'Université de Fribourg, on fait comme s'il était possible de parler des langues différentes sans développer également des significations et des références culturelles différentes. Quel tollé et quelle incompréhension si, au lieu de se revendiquer "I'Université bilingue ", l'Université de Fribourg se revendiquait "l'Université biculturelle »! L'Université de Fribourg est ainsi un bel exemple du deuxième cas de figure de combinaison entre diversité des langues et des cultures, tout comme l'est d'ailleurs le cas suivant.

\section{Deuxième cas : une association scientifique, nationale et plurilingue}

Il s'agit ici non plus d'une institution ancrée dans un contexte local, mais d'un réseau de chercheurs, intéressés aux approches interculturelles de l'éducation et qui vivent dans les différentes régions linguistiques de la Suisse (à vrai dire, principalement en Suisse romande, francophone, et en Suisse alémanique, germanophone). Ce réseau, appelé " Groupe de travail Éducation interculturelle de la Société Suisse de Recherche en Éducation (SSRE) », dont j'assure la coordination depuis quelques années, sert de lieu d'échange d'informations et organise parfois des rencontres scientifiques. Avec quelque peine, car il est malheureusement difficile de mobiliser ensemble les membres des différentes régions linguistiques. 
Comme il est habituel en Suisse, ce groupe plurilingue fonctionne sur le principe de l'intercompréhension, en allemand, français et italien (celui-ci étant en réalité très peu utilisé, le groupe de travail ne comptant que de très rares membres de la Suisse italophone). Il faut bien l'avouer, il n'y a pas que le manque de disponibilité qui puisse expliquer la difficulté à mobiliser les membres de ce groupe de travail pour des activités communes. Loin du stéréotype des Suisses polyglottes, il s'avère que seule une minorité de chercheurs se sent à l'aise dans la pratique de l'intercompréhension en français et allemand, et encore moins si on y ajoute l'italien. Et ceci alors que la plupart ont fait leur scolarité en Suisse et ont donc étudié au moins deux langues nationales. Un recensement de la recherche en éducation interculturelle en Suisse, réalisé par plusieurs membres - francophones - de ce groupe de travail, avait ainsi montré d'importantes différences dans la recherche pratiquée en Suisse romande et en Suisse alémanique (Ogay et collab., 2008). Cela se retrouve également dans les références bibliographiques que nous utilisons dans nos publications: les chercheurs de Suisse alémanique se réfèrent aux publications allemandes, et leurs collègues de Suisse romande aux publications françaises, ce n'est que sur les publications en anglais que nous nous retrouvons (et encore). Les chercheurs travaillant sur l'interculturalité auraient-ils de la difficulté à la mettre en pratique?

\section{Troisième cas : une organisation non gouvernementale de jeunesse, au niveau international et national}

Des quatre cas que j'ai choisi d'examiner ici, celui-ci est le seul qui relève d'une expérience passée. II s'agit de mon activité de bénévole (au niveau romand, puis national et international) dans une organisation de jeunesse, ICYE (International Cultural Youth Exchange), permettant à des jeunes de tous les continents de passer une année d'échange dans un autre pays.

Au niveau international, on y retrouve la troisième comme la quatrième des combinaisons entre diversité culturelle et linguistique identifiées précédemment: dans les échanges formels, la communication se passait dans la langue officielle de l'association, l'anglais, qui permettait ainsi à ces personnes venant de contextes linguistiques et culturels très divers de communiquer dans une langue commune. Mais il n'était pas rare dans les moments informels d'assister à ce " tout pluri » de la quatrième dimension qui combine communication interculturelle et plurilingue. J'ai ainsi pu faire l'expérience des apports et difficultés d'une lingua franca, qui permettait de communiquer certes mais qui provoquait également passablement de flou dans la compréhension car très peu la maîtrisaient réellement. J'ai aussi expérimenté dans les moments informels la richesse et la complexité d'une pratique plurilingue, qui permettait de réunir des personnes parlant différentes langues, mais qui excluait aussi ceux qui ne partageaient pas les langues utilisées (typiquement, les anglophones, favorisés dans les situations formelles, étaient souvent démunis dans les moments informels plurilingues). Quant à mon expérience dans l'association au niveau national, qui en théorie devait correspondre au deuxième cas de figure identifié (références culturelles supposément communes et langues différentes), elle m’a plongée dans une grande perplexité qui m’a amenée quelques années plus tard à ma recherche de doctorat sur les relations entre Suisses romands et Suisses alémaniques (Ogay, 2000) : comment se pouvait-il qu'autant de bénévoles de Suisse romande, ayant effectué une année d'échange dans un pays étranger et montrant une grande ouverture interculturelle, soient si réticents à participer à des activités au niveau national avec leurs collègues alémaniques? Dans ce cadre également, la pratique de l'intercompréhension en allemand et français semblait difficile pour beaucoup. Fort heureusement, les langues apprises pendant l'année d'échange permettaient de nouvelles recompositions linguistiques réunissant Romands et Alémaniques, mais ces nouveaux regroupements entre hispanophones, anglophones, finnophones (?) étaient finalement autant de nouvelles divisions. On s'en doute, la réticence de mes collègues bénévoles romands à prendre part aux activités avec les collègues alémaniques n'était pas due à la seule question linguistique, tant s'en faut. II n'était pas difficile d'observer dans leurs propos l'expression de préjugés négatifs envers les compatriotes alémaniques, tels qu'ils ont pu être relevés dans différents travaux sur les relations entre Romands et Alémaniques, notamment par Windisch (1992). C'est ainsi que je fis mes premières tentatives de formation interculturelle, cherchant à travailler sur les préjugés entre Romands et Alémaniques, inspirée par les travaux réalisés dans le cadre de l'OFAJ (Office franco-allemand pour la jeunesse, 1987).

\section{Quatrième cas : une association scientifique, internationale et monolingue}

Le dernier contexte est celui de I'ARIC, Association pour la Recherche Interculturelle, qui réunit des chercheurs de divers pays et dont la langue de communication voulue par les fondateurs est le français, une configuration qui

Alterstice - Revue Internationale de la Recherche Interculturelle, vol. 2, $n^{\circ} 1$ 
correspond au troisième cas de figure identifié plus haut. L'ARIC se distingue de la plupart des associations internationales qui, comme ICYE ci-dessus, adoptent plus volontiers l'anglais comme langue de communication. La grande majorité des membres de I'ARIC provenant de pays ou de régions francophones (principalement de Suisse romande, du Québec et de France), le français est généralement leur langue première. Ces dernières années cependant, un nombre important de collègues venant de pays non francophones ont rejoint l'association. Cette internationalisation de I'ARIC a suscité une remise en question parfois houleuse du français comme seule langue de communication de l'association. La langue française a en effet changé de statut: de langue première partagée par tous (sans ignorer bien sûr les variations régionales parfois importantes), le français est passé au statut de lingua franca, code en principe commun mais diversement familier aux uns et aux autres. Cependant, certains membres venant de contextes non francophones n'adhèrent pas à la solution du français comme lingua franca et ont exprimé le souhait de pouvoir également utiliser leurs propres langues au sein de I'ARIC. Cette demande revient à remplacer la communication interculturelle via une lingua franca (la troisième combinaison) par une communication interculturelle et plurilingue (la quatrième combinaison).

\section{Perspective comparée, quelques pistes de réflexion}

Ces quatre cas nous présentent différentes combinaisons entre pluralité des langues et des cultures, passant souvent de l'une à l'autre. Ils nous interrogent sur leurs articulations complexes et les solutions qui peuvent être trouvées pour assurer la communication. Est-il possible de se comprendre, c'est-à-dire de développer une culture commune, tout en parlant des langues différentes? Si l'exemple de la Suisse - comme des nombreux autres États plurilingues ou, même, de l'Union européenne - indique que cela est possible, il montre aussi combien cette identité commune qui ne bénéficie pas d'une langue partagée est fragile et demande des efforts constants, de la part des institutions comme des individus (pour la Suisse, voir Kreis, 1994). D'un autre côté, peut-on parler une même langue sans que celle-ci soit l'imposition d'une culture, mais qu'au contraire malgré cette langue commune des références culturelles diverses puissent se maintenir et s'exprimer?

La comparaison des différentes combinaisons entre diversité linguistique et culturelle que représentent les cas exposés ci-dessus soulève les points suivants :

- Dans notre réflexion sur les pratiques et les politiques linguistiques, il est important de distinguer le niveau institutionnel et le niveau des individus. L'institution peut définir sa politique linguistique et reconnaître une ou plusieurs langues comme langues de communication dans l'institution. Mais les compétences linguistiques des individus ne recouvrent pas nécessairement ce qui est prescrit au niveau institutionnel, et leurs pratiques peuvent diverger plus ou moins de la norme institutionnelle, selon notamment le caractère plus ou moins formel et public de la situation d'interaction. Ainsi, l'Université de Fribourg est institutionnellement bilingue allemand-français (comme le groupe de travail de la SSRE, ou ICYE au niveau suisse), mais ses membres ne pratiquent pas nécessairement ce bilinguisme, ils sont peut-être monolingues, ou alors ils pratiquent d'autres langues que les langues officielles. De son côté, I'ARIC peut être officiellement monolingue, francophone, mais ses membres viennent d'horizons linguistiques de plus en plus divers et de nombreuses autres langues sont entendues dans les échanges informels.

- Dans la gestion de l'articulation entre diversité des langues et diversité des cultures, seule la dimension linguistique peut faire l'objet d'une politique, de prescriptions institutionnelles mais aussi, dans une certaine mesure, à destination des individus. Il est possible de décider du code linguistique qui est utilisé dans la communication (même si la norme est ensuite livrée à l'interprétation individuelle), il ne peut en être de même pour les références culturelles que les interlocuteurs apportent et négocient plus ou moins consciemment dans leurs interactions. La réponse pour la gestion de l'articulation entre diversité des langues et des cultures se trouve ainsi au niveau de la politique linguistique adoptée, politique qui va plus ou moins permettre ou contraindre l'expression de la diversité culturelle.

- Un contexte plurilingue peut paradoxalement renforcer la séparation entre les groupes linguistiques et, par là, leurs différences culturelles : j'ai pu observer à maintes reprises, même dans des contextes qui officiellement valorisent la diversité des langues et des cultures, que nous avons tous tendance à nous regrouper par ressemblance, principalement sur le plan linguistique et/ou culturel (ce que les psychologues sociaux appellent l'attraction par la similitude, Byrne, 1997). Dans un contexte plurilingue, sans lingua franca 
reconnue voire imposée, s'il n'y a pas une volonté institutionnelle claire et un travail intense de s'opposer à cette tendance au regroupement par similitude et de travailler le contact et l'échange, les individus se regroupent par langues. Les échanges qu'ils ont entre eux leur permettent ainsi de développer ou de renforcer une culture commune, dans le sens de significations partagées, ce qui a pour conséquence d'augmenter la similitude intragroupe mais aussi la différence intergroupe. Il ne suffit ainsi pas d'avoir un contexte plurilingue pour que les individus s'aventurent dans la communication plurilingue. J'ai pu l'observer dans les trois contextes officiellement bilingues que sont l'Université de Fribourg, le groupe de travail de la SSRE et ICYE au niveau suisse. J'ai même pu l'observer au sein de la supposée monolingue ARIC, lors des moments informels où l'on voit les personnes se regrouper par affinités linguistiques.

- Le plurilinguisme peut masquer la question de la diversité des cultures : comme on l'a vu, toute l'attention en Suisse est portée sur la diversité linguistique, l'idée selon laquelle il y aurait des différences culturelles entre les groupes linguistiques est un tabou. La diversité culturelle représente en effet un risque pour l'harmonie confédérale entre les quatre régions linguistiques et les 26 cantons, une harmonie dont la création puis le maintien ont requis d'importants efforts au cours de I'histoire (Im Hof, 1991). Quelle attention recevrait la diversité culturelle dans une organisation internationale comme I'ARIC si celle-ci abandonnait sa lingua franca, le français, et faisait le choix du multilinguisme? L'enjeu de la compréhension plurilingue, tellement difficile à gérer ne serait-ce que sur un plan pratique, risquerait de reléguer aux oubliettes celui de la compréhension interculturelle.

- La pratique de l'intercompréhension ne va pas de soi, et ceci même lorsque les langues concernées sont définies et censées être connues des individus : comme j'en ai fait l'expérience à l'Université de Fribourg, au sein du groupe de travail de la SSRE comme de l'association de jeunesse ICYE au niveau suisse, la règle du " chacun parle sa langue » est loin d'être évidente, même lorsqu'il n’y a que deux langues concernées, dans ces contextes l'allemand et le français, et que chacun ou presque a derrière lui plusieurs années d'apprentissage scolaire de la langue dite partenaire. J'ai peine à imaginer ce que cela donnerait dans un contexte international comme celui de l'ARIC, où les langues potentielles qu'il faudrait « intercomprendre » sont innombrables. Ou plutôt, il peut être très facile d'imaginer ce qui se passerait : I'anglais serait rapidement adopté comme lingua franca, comme cela est si souvent le cas, même en Suisse aujourd'hui. La difficulté de l'intercompréhension, stratégie à première vue très attrayante, c'est qu'il faut être prêt à accepter et assumer que le principe de "chacun parle sa langue " ne signifie pas seulement "je peux parler ma langue ", mais aussi « je comprends les langues des autres », ce qui est bien plus difficile.

\section{Tension dialectique et recherche d'équilibre dans l'articulation entre langues et cultures}

Les pratiques de gestion de la diversité linguistique et culturelle dans ces différents contextes, aux histoires et contraintes différentes, sont autant de tentatives de trouver un équilibre dans la tension dialectique entre l'égalité et la diversité (Ogay et Edelmann, 2011). La difficulté est que cette tension s'exerce sur deux plans : au niveau des langues comme des cultures. Entre les deux extrêmes que sont la communication monolingue et monoculturelle et la communication multilingue et multiculturelle, quelle combinaison entre diversité linguistique et culturelle est la plus favorable à la communication? Comme on ne peut - et encore - que décider du code linguistique utilisé et non pas des références culturelles apportées par les interlocuteurs dans l'interaction, la réponse tourne autour des langues que l'on adopte dans un certain contexte. Une pétition de principe pour la diversité est certainement séduisante pour qui travaille "dans l'interculturel », l'analyse des quatre cas présentés montre qu'une telle pétition, aussi généreuse soit-elle, risque au final de nuire au dialogue.

Si je reviens à la question de la ou des langues officielles au sein d'une association internationale comme l'ARIC, mon expérience dans les trois contextes supposés bilingues examinés plus haut m'amène à penser qu'il est très ambitieux pour une institution de vouloir amener les individus à une pratique plurilingue. C'est le choix exigeant fait par l'Union européenne, qui cherche en particulier à développer l'intercompréhension entre langues romanes (Degache, 2005). Mais les moyens de l'Union européenne sont tout autres que ceux dont dispose une modeste association comme I'ARIC. D'autant plus que le bassin de recrutement de I'ARIC n'est pas le seul continent européen, mais qu'il est potentiellement mondial. Si les références culturelles des membres de l'ARIC sont si diverses, il semble peu raisonnable d'y ajouter une telle diversité des codes linguistiques pour les exprimer.

Alterstice - Revue Internationale de la Recherche Interculturelle, vol. 2, $n^{\circ} 1$ 
Le maintien du français comme langue officielle de l'association semble dès lors le seul choix raisonnable. Cependant, le questionnement apporté par les collègues pour qui le français n'est pas la langue première met en évidence que l'usage du français comme langue de communication ne peut plus se faire comme du temps de la fondation de l'association par des francophones, pour qui communiquer en d'autres langues n'aurait pas eu beaucoup de sens. D'un usage du français comme une évidence partagée, il s'agit de passer à un usage négocié du français, en contact avec d'autres espaces linguistiques et culturels. Il y a pour cela un certain nombre de conditions que les francophones de l'association - représentant pour l'instant le groupe majoritaire - se doivent de respecter vis-à-vis des collègues pour qui le français est une langue étrangère.

Il s'agit notamment de se rappeler que la communication n'a pas pour seul objectif la transmission d'information mais qu'elle est également un lieu de négociation d'identités, personnelles et sociales. Les identités culturelles et linguistiques des membres venant de nouveaux horizons ont probablement été insuffisamment reconnues dans I'ARIC. Comme le montre la théorie de l'accommodation de la communication (Gallois, Ogay et Giles, 2005), un locuteur sera prêt d'adopter le code linguistique d'un autre locuteur (accommodation par la stratégie de la convergence) dans la mesure où il pense que cela facilitera la communication (motivation cognitive, ou instrumentale) mais aussi que cela ne menace pas son identité, personnelle et sociale. Si au contraire il y a un sentiment de menace de l'identité, alors ce locuteur adoptera la stratégie de divergence, afin de se différencier, et sera réticent à parler la langue de l'autre. Des blessures et des revendications identitaires se cachent probablement derrière la remise en question du français comme langue de travail au sein de l'ARIC. En particulier, la langue française traîne derrière elle le lourd passé colonial de l'État français (voir Lehmil, 2006), qui influence très certainement la façon dont elle est perçue notamment dans les pays qui ont subi cette colonisation. Or cet héritage n'est pas celui des Suisses, Belges et Canadiens francophones, qui ne s'y identifient pas et oublient souvent que c'est avec cette clé de lecture que leur langue est perçue.

Paradoxalement, accorder toute l'attention nécessaire aux dimensions identitaires qui se jouent dans la communication en français entre personnes qui s'identifient à cette langue et personnes qui s'identifient à d'autres langues signifie qu'il s'agit de donner à la langue française comme lingua franca le seul rôle d'un outil de communication. II s'agit d'expliciter sa fonction instrumentale et de relativiser la dimension culturelle (qui prend des formes très différentes selon que l'on se trouve en France ou dans d'autres pays francophones) : I'ARIC n'est pas une communauté fondée sur la langue française comme expression d'une culture commune, mais une association qui utilise cette langue, car il en faut bien une. Il est vrai que le choix du français par les fondateurs de I'ARIC était effectivement une revendication identitaire, non pas française mais francophone, refusant la domination de l'anglais sur les échanges scientifiques internationaux. Aujourd'hui, faire de la langue française une langue de communication internationale implique d'accepter - comme ont dû le faire les Britanniques avec l'anglais international - que le français dans ce contexte devienne un sabir qui appartient à tous ceux qui le parlent, une langue qui connaît son évolution propre par rapport au français des francophones de France.

\section{Conclusion}

Trouver la bonne articulation entre pluralités linguistique et culturelle n'est assurément pas une sinécure, tant ces deux dimensions de la diversité humaine sont interdépendantes, se nourrissant l'une l'autre mais sans pour autant se recouvrir. Trois des contextes institutionnels examinés ici nous montrent que la communication bilingue, et $a$ fortiori plurilingue, est loin d'être une évidence et ne peut simplement se décréter en comptant sur la seule bonne volonté des individus (à moins de rester élitaire). De plus, le plurilinguisme n'encourage pas nécessairement la reconnaissance de la diversité culturelle, tant s'en faut: l'effort investi dans la compréhension de plusieurs codes linguistiques peut être tel que les interlocuteurs n'ont pas en plus la disponibilité suffisante pour être attentifs aux références culturelles des uns et des autres. D’un autre côté, la question soulevée par les membres non francophones de I'ARIC au sujet du français comme seule langue de communication permet de rappeler les risques

de l'illusion de similitude que peut produire le fait de s'exprimer dans une langue commune alors que les références culturelles sont différentes et que le rapport - également historique - à cette langue est différent. 
Comme dans toute chose peut-être, cette articulation de la pluralité des langues et des références culturelles est une affaire d'équilibre à trouver - et à entretenir continuellement - entre l'égalité (le partage, la similitude) et la diversité. Si dans la recherche interculturelle nous sommes bien conscients des ravages du "mono ", il s'agit de veiller à ne pas verser dans les illusions du " tout multi », qui de plus est sur le plan des langues ET des cultures. Un certain pragmatisme me semble nécessaire pour garantir la possibilité de la communication: I'expression de la diversité culturelle a quand même besoin d'un certain partage des codes linguistiques utilisés, et c'est en cela que la dimension culturelle se trouve conditionnée par la dimension linguistique.

\section{Références bibliographiques}

Byrne, D. (1997). An overview (and underview) of research and theory within the attraction paradigm. Journal of Social \& Personal Relationships, 14(3), 417-431.

Capucho, F. (2008). L'intercompréhension est-elle une mode? Du linguiste citoyen au citoyen plurilingue. PRATIQUES, 139/140, 238-250.

Degache, C. (2005). Comprendre la langue de l'autre et se faire comprendre ou la recherche d'une alternative communicative: le projet Galanet. SYNERGIES-Italie. Approches pédagogiques et instruments didactiques pour le plurilinguisme, 2, 50-60.

Duquette, G. (2004). Les différentes facettes identitaires des élèves âgés de 16 ans et plus inscrits dans les écoles de langue française de l'Ontario. Francophonies d'Amérique, 28, 77-92.

Gallois, C., Ogay, T. et Giles, H. (2005). Communication Accommodation Theory: a look back and a look ahead. Dans W. Gudykunst (dir.), Theorizing about intercultural communication (p. 121-148). Thousand Oaks : Sage.

Gohard-Radenkovic, A. (2007). Le statut du français dans une Suisse à quatre langues : représentations et paradoxes. Revue japonaise de didactique du français, 2(2), 43-59.

Grin, F. (2010). L'aménagement linguistique en Suisse. Télescope, 16(3), 55-74.

Im Hof, U. (1991). Mythos Schweiz. Identität - Nation - Geschichte 1291-1991. Zürich : Verlag Neue Zürcher Zeitung.

Kreis, G. (1994). La Suisse chemin faisant. Rapport de synthèse du Programme national de recherche 21 « Pluralisme culturel et identité nationale ». Lausanne: L'Âge d'Homme.

Lehmil, L. (2006). L'édification d'un enseignement pour les indigènes : Madagascar et l'Algérie dans l'Empire français. Labyrinthe, 24(2), 91-112. http://labyrinthe.revues.org/1252

Lüdi, G. (2006). De la compétence linguistique au répertoire plurilingue. Bulletin suisse de linguistique appliquée, $84,173-189$.

Office franco-allemand pour la jeunesse. (1987). Promouvoir les apprentissages interculturels : réflexions à propos d'une formation des animateurs et des responsables des programmes de rencontres. Textes de travail, 7. http://www.ofaj.org/paed/texte2/formanim/formanim.html

Ogay, T. (2000). De la compétence à la dynamique interculturelles. Des théories de la communication interculturelle à l'épreuve d'un échange de jeunes entre Suisse romande et alémanique. Berne : Peter Lang.

Ogay, T., et Edelmann, D. (2011). Penser l'interculturalité dans la formation des professionnels : I'incontournable dialectique de la différence. In A. Lavanchy, F. Dervin et A. Gajardo (dir.), Anthropologies de l'interculturalité. (p. 47-71). Paris : L'Harmattan.

Ogay, T., Zharkova Fattore, Y., Borruat, S., Gakuba, T., Gremion, M. et Hutter, V. (2008). La recherche empirique en éducation interculturelle en Suisse : comparaison entre la Suisse francophone et la Suisse germanophone. Revista Española de Educación Comparada, 14, 177-197.

Windisch, U. (1992). Les relations quotidiennes entre Romands et Suisses allemands : les cantons bilingues de Fribourg et du Valais. Lausanne : Payot.

Alterstice - Revue Internationale de la Recherche Interculturelle, vol. 2, $n^{\circ} 1$ 